\title{
Regulation of Brain Pyruvate Dehydrogenase Multienzyme Complex
}

\author{
T. T. NGO AND A. BARBEAU
}

SUMMARY: A number of excellent and comprehensive reviews on various as. pects of pyruvate dehydrogenase multienzyme complex have been written recently. The purpose of the present review is to summarize briefly the reaction mechanism and the regulation of this enzyme. Emphasis is put on the most recent literature not covered by previous reviews. Particular attention is also paid to the regulation of brain pyruvate dehydrogenase multienzyme complex, since a number of patients with neuromuscular diseases, such as Friedreich's ataxia, show a decreased rate of pyruvate oxidation.

RÉSUMÉ: Un grand nombre de revues complètes sur les différents aspects du complexe multienzymatique de la pyruvate deshydrogénase (PDH) ont été récemment publiés. Le but du présent travail sera de résumer certains points nouveaux concernant le mécanisme de la réaction et de la régulation de ce complexe enzymatique dont l'importance grandissante apparaît dans l'étude de plusieurs maladies musculaires, dont l'ataxie de Friedreich, qui toutes démontrent un ralentissement $d u$ taux d'oxydation du pyruvate.

From the Department of Neurobiology, Clinical Research Institute of Montreal.

Reprint requests to: Dr. A. Barbeau, Clinical Research Institute of Montreal, 110 Pine Avenue West, Montreal, Quebec, Canada H2W 1R7.

\section{REACTION MECHANISM}

The oxidative decarboxylation of pyruvate to form acetyl $\mathrm{CoA}$ and $\mathrm{CO}_{2}$ at the expense of NAD is catalyzed by pyruvate dehydrogenase $(\mathrm{PDH})$. In all organisms studied, PDH is a multienzyme complex. In bacteria, it consists of 3 distinct enzymes: (1) pyruvate decarboxylaste $\left(E_{1}\right)$ (also called pyruvate dehydrogenase); (2) dihydrolipoate transacetylase $\left(\mathrm{E}_{2}\right)$; and (3) dihydrolipoate dehydrogenase $\left(E_{3}\right)$. These enzymes catalyze the following reactions (reactions 1 to 5 ) in a ssequential manner (Table I).

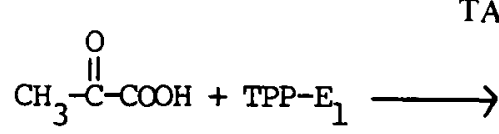

\section{TABLE I}

(2)
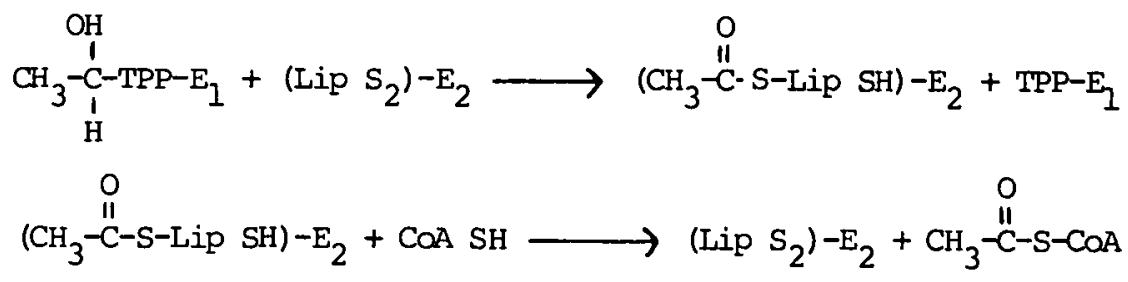

(4)

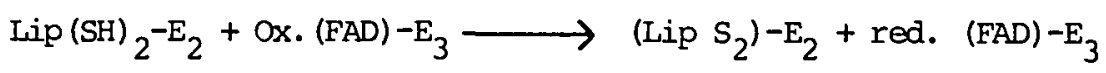

(5) Red. (FAD) $-\mathrm{E}_{3}+\mathrm{NAD}^{+} \longrightarrow$ ox. (FAD) $-\mathrm{E}_{3}+\mathrm{NADH}+\mathrm{H}^{+}$

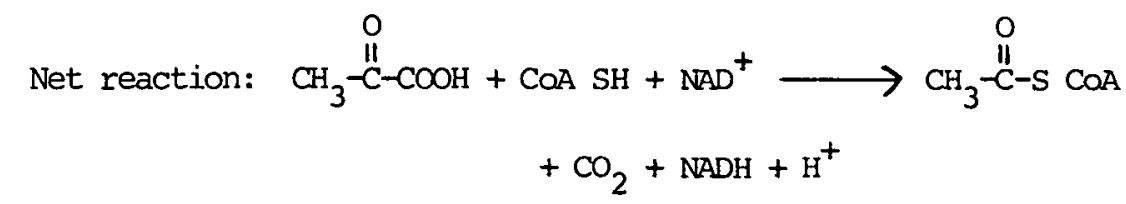

E1 catalyzes reactions 1 and $2, E_{2}$ catalyzes reaction $3, \mathrm{E}_{3}$ catalyzes reactions 4 and $5 . E_{2}$ forms the core of the complex, and $E_{1}$ and $E_{3}$ are attached to $E_{2}$. The lipoyl group is bound covalently to $E_{2}$ via an amide linkage to the $\varepsilon$-amino group of a lysyl residue in $E_{2}$. This attachment provides a flexible arm, permitting the lipoyl group to rotate between the catalytic centers of the three associated enzymes which form the pyruvate dehydrogenase multienzyme complex (Reed, 1960, 1974; Hucho, 1975; Denton et al., 1975).

The detailed chemical mechanism of the pyruvate dehydrogenase catalyzed reactions having been reviewed by Hucho (1975), only a very brief survey will be given here. A schematic representation of the reaction sequence of $\mathrm{PDH}$ is shown in Figure 1. 


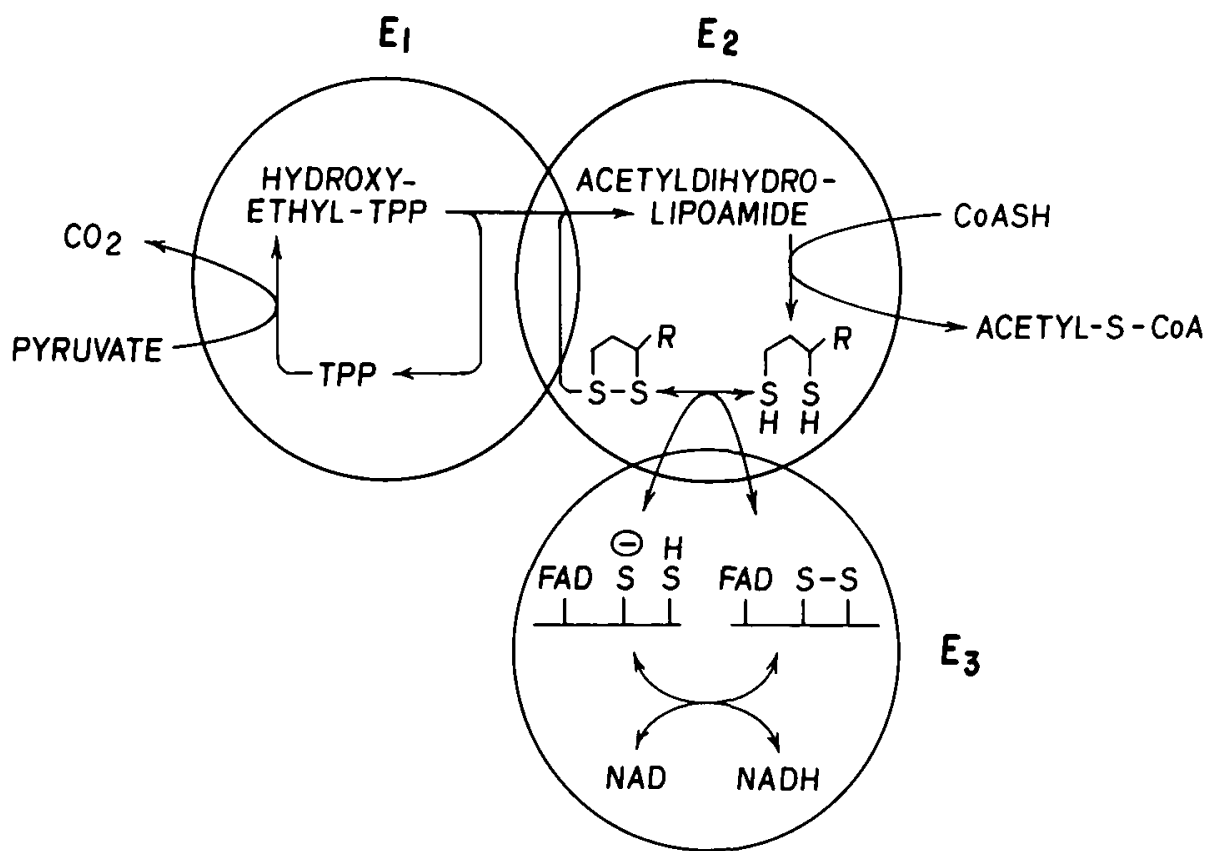

Figure 1 -Sequence of the reaction catalyzed by pyruvate dehydrogenase multienzyme complex $(\mathrm{PDH}) . \mathrm{TPP}=$ thiamine pyrophosphate; $\widetilde{S}_{\mathrm{S}} \mathrm{S}^{\mathrm{N}}=$ oxidized lipoamide: $\mathrm{H}_{\mathrm{H}^{+}}^{\mathrm{S}_{\mathrm{H}^{+}}}{ }^{+}$ reduced lipoamide. $\mathrm{CoA} \mathrm{SH}=$ coenzyme $\mathrm{A} ; \mathrm{FAD}=$ flavin adenine dinucleotide; $\mathrm{NAD}=$ nicotinamide adenine dinucleotide; $\mathrm{NADH}=$ reduced form of nicotinamide adenine dinucleotide; $E_{1}=$ pyruvate decarboxylase; $E_{2}=$ dihydrolipoamide transacetylase; $E_{3}=$ dihydrolipoamide dehydrogenase.

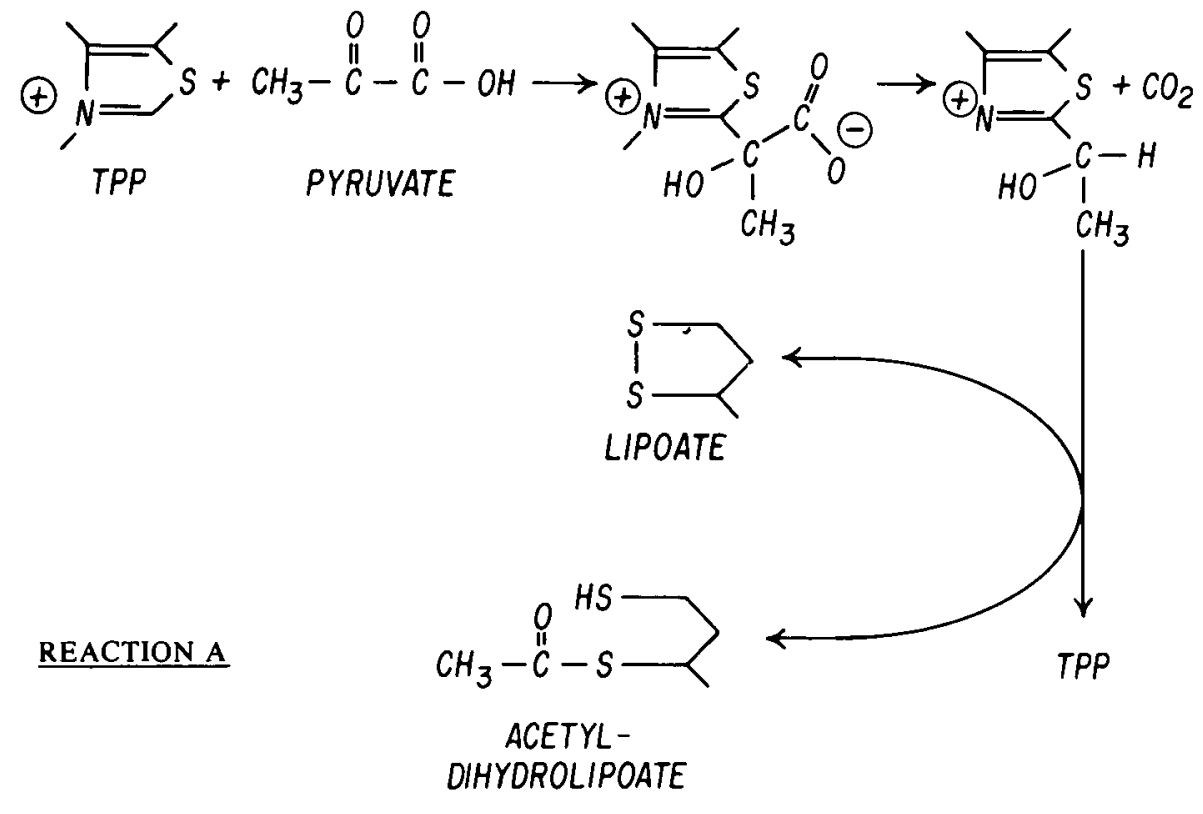

E1 catalyzes 2 reactions: 1) the decarboxylation of pyruvate which is initiated by its attachment to the 2-position of the thiazole ring of TPP. The loss of $\mathrm{CO}_{2}$ leads to hydroxyethyl-TPP. Magnesium ion is the co-factor of $E_{1} ; 2$ ) the oxidation of the hydroxyethyl group and transfer of the resulting acetyl group to lipoic acid of $E_{2}$. Thus, in addition to the decarboxylating activity, $E_{1}$ also possesses dehydrogenase and transferase activities. The reaction catalyzed by $E_{1}$ is irreversible. The reaction sequence catalyzed by $E_{1}$ can be summarized as:

$E_{2}$ catalyzes the transfer of the acetyl group of the dihydrolipoic acid to CoA SH. This reaction is reversible.

$E_{3}$ catalyzes the regeneration of active $E_{2}$ making it possible for the next catalytic cycle of the complex. Regeneration of $E_{2}$ occurs by the transfer of two electrons from dihydrolipoic acid to NAD, a reaction in which two molecules of NAD, one molecule of FAD, and one disulfide group of the protein take part. Thus, functionally, $E_{3}$ can be depicted as:

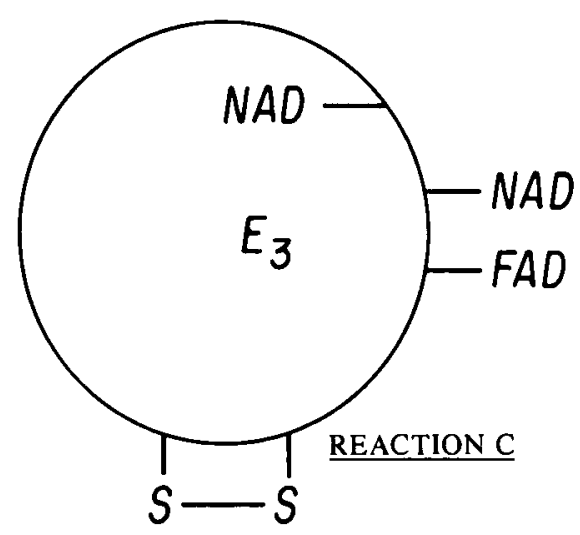

These chemical evidences of the reaction mechanism suggest that the over-all reaction catalyzed by pyruvate dehydrogenase proceeds via a three-site "ping-pong" mechanism. Indeed, kinetic studies of Tsai, Burgett and Reed (1973) and of Ngo and Barbeau (J. Neurochem., in press, 1978) have provided evidence that both bovine kidney and rat brain pyruvate dehydrogenase multienzyme complexes catalyze the reaction via a three-site "ping-pong" mechanism. 


\section{METABOLIC REGULATION}

Pyruvate occupies a strategic position in the metabolism of both carbohydrates and lipids. Any enzyme that catalyzes the metabolism of pyruvate is expected to be under strict metabolic regulation. The complexity of the control mechanism arises from the position of pyruvate and pyruvate metabolizing enzyme in energy metabolism. This enzyme is at the crossroads of several metabolic routes. Pyruvate is formed in glycolysis from glucose, from lactate, and from amino acids such as alanine and cysteine. Concurrently, it is the starting point of four important metabolic routes (Figure 2):

1. Pyruvate can be oxidatively degraded to acetyl CoA and NADH which finally, through the tricaboxylic acid cycle and via oxidative phosphorylation, can generate $A$ TP.

2. Pyruvate can also be carboxylated to form oxolacetate and thus can form the starting point of gluconeogenesis.
3. The acetyl CoA formed from oxidative decarboxylatation of pyruvate can be used for the starting material in the biosynthesis of fatty acids, prostaglandins and steroidal hormones.

4. Pyruvate supplies the carbon skeleton for the de novo synthesis of alanine and cysteine.

The apparent activity of an enzyme can be regulated in many ways. Enzyme activity can be modulated by extracellular agents such as hormones or their second messengers, i.e., cyclic nucleotides. Intracellularly, an enzyme activity can be controlled by the following processes:

\section{A) Coarse control mechanism}

A coarse control for enzyme activity means a long term regulation of enzyme activity by changing the concentration of enzyme. The enzyme level of cells can be increased by induction (derepression) or decreased by repression. The processes of enzyme induction and repression in the cells of multicellular or-
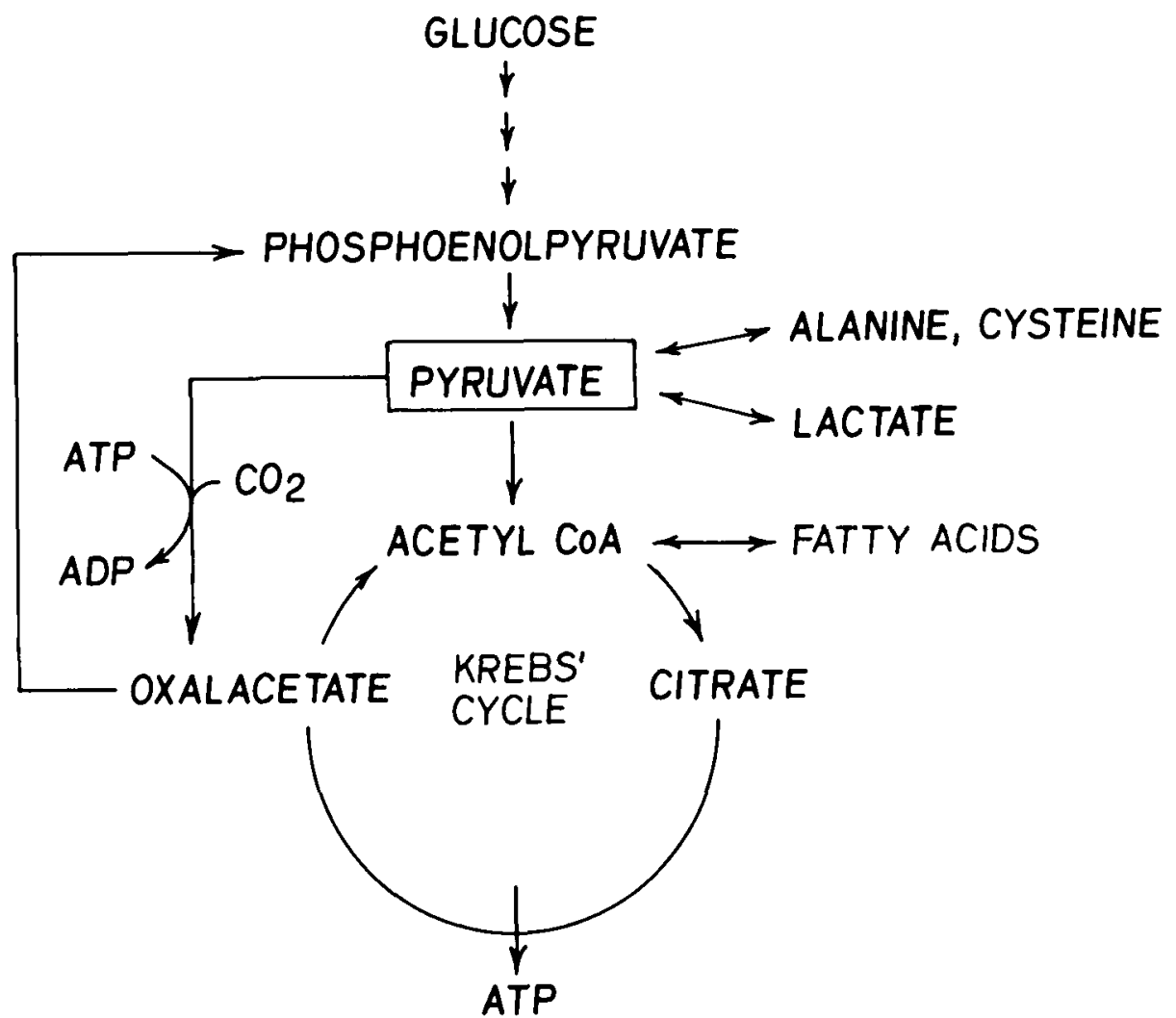

Figure 2-Pathways of pyruvate metabolism showing the strategic position of pyruvate at the crossroads of carbohydrate and lipid metabolisms.

ganisms differ from that of unicellular organisms. The regulatory mechanisms that have evolved in bacteria are geared to achieve a maximal rate of cell proliferation consistent with available nutritional sources. In contrast the individual cell of a multicellular organism more often divides slowly, and is variable in degree of organisational complexity and in the type a specific function it carries out. Such cells are associated with both similar and dissimilar cells into tissues and organs, functioning as an integrated whole in the protection and propagation of specialized cells. In bacteria, the removal process can involve dilution during phases of rapid growth. In animal tissues, where little cellular division takes place, the process of protein degradation becomes increasingly significant as a means of removing unneeded metabolic machinery, and as a means for controlling enzyme levels.

The interaction between the synthesis and degradation of specific enzymes in controlling enzyme levels in animal tissues is reflected in a general comparison of differences in the time course of enzyme induction in animal tissues and bacteria (Figure 3). In bacteria the total enzyme activity increases, following the introduction of an inducer. Similarly, in animal cells the level of enzyme can be increased by induc-

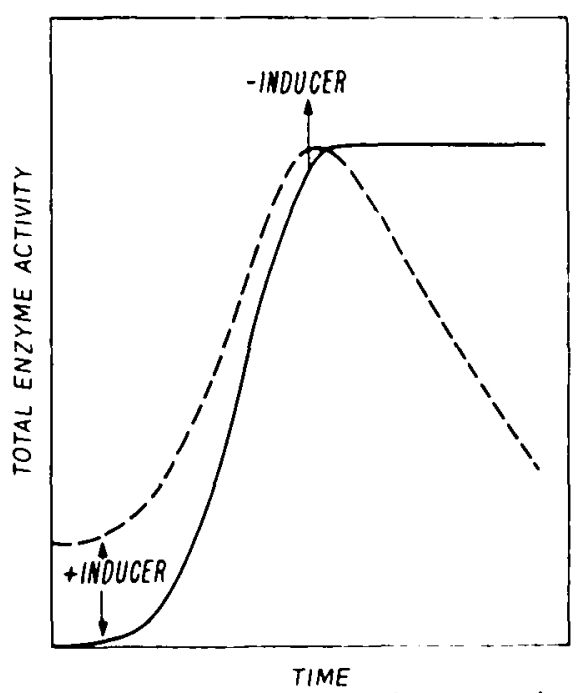

Figure 3-Time courses of enzyme induction in bacteria (solid line) and animal cells (broken line). 
ers such as hormones, substrate, or changes in diet. However, in contrast to the sustained level of the induced enzymes in bacteria, in mammalian cells the induced enzyme activity returns to a basal level along an exponential time course once the inducer is removed (Schimke, 1969; Changeux, 1965).

In $E$. coli the synthesis of the pyruvate dehydrogenase multienzyme complex is subject to genetic regulation. It can be induced by pyruvate. The genetic control of this multienzyme complex in mammalian cells in not clearly understood. Difficulties in obtaining appropriate mutants of mammalian cells have hampered our understanding of the genetic control of the synthesis of pyruvate dehydrogenase multienzyme complex. Blass et al. (1970) observed a defect in pyruvate decarboxylase in a child with an intermittent movement disorder. Pyruvate decarboxylase is the first enzyme in the coordinated reactions of pyruvate dehydrogenase multi-enzyme complex. Subsequently, Kark et al. (1974) showed that patients with spinocerebellar degeneration and motor neuropathies oxidized pyruvate in their muscles significantly more slowly than the controls with myopathic disease or normal muscle. Impaired pyruvate oxidation in the anterior cerebellar vermis by deficiencies of pyruvate dehydrogenase, which would be too mild to affect pyruvate oxidation in other areas of the brain, has been postulated as a possible mechanism for selective cerebellar damage in partial pyruvate dehydrogenase deficiency (Reynolds and Blass, 1976). Low activities of the pyruvate and oxoglutarate dehydrogenase multienzyme complexes in fibroblasts of patients with Friedreich's ataxia were recently reported by Blass et al. (1976). Barbeau (1975) has also observed a decrease in pyruvate oxidation and an increase in the level of both pyruvate and lactate in the blood of Friedreich's ataxia after glucose loading. Recent findings indicate that the most likely cause of this decrease is an impaired functioning of lipoamide dehydrogenase, the terminal enzyme common to both pyruvate and oxoglutarate dehydrogenase multienzyme complexes (Blass et al., 1976; Barbeau et al., 1976). Furthermore, we have observed that rat brain pyruvate dehydrogenase multienzyme complex is inactivated by pyridoxal phosphate, a coenzymatically active form of Vitamin B6. In addition, we also observed that the putative neurotransmitter, taurine, strongly activates the pyridoxal phosphate inactivated enzyme. The mechanisms of pyridoxal phosphate inactivation and taurine reactivation of the inactivated enzyme are currently being investigated.

Slowing down of pyruvate oxidation in patients with neurologic diseases will cause an abnormal metabolism of carbohydrates and lipids. This in turn leads to impaired functioning of the brain, since the brain depends on oxidative metabolism for its proper function (Mcllwain and Bachelard, 1971).

\section{B) Fine control mechanism}

Fine control mechanisms refer to the direct control of any enzyme catalytic activity by a modifier. Thus the enzyme activity can be switched on or off almost instantaneously.

1. Product inhibition. The activity of pig and rat brain PDH is competitively inhibited by acetyl $\mathrm{CoA}$, a reaction product of $\mathrm{PDH}$ ( $\mathrm{Ngo}$ and Barbeau, J. Neurochem., in press, 1978; Siess et al., 1971). Furthermore, we have shown that NADH also competitively inhibits rat brain PDH with respect to NAD. These observations suggest that the activity of PDH may be regulated in vivo by acetyl $C o A$ and $C O A$ ratio and the oxidation state of the cell. The sites of acetyl CoA and NADH inhibition are the dihydrolipoate transacetylase and the lipoamide dehydrogenase components of the complex, respectively.

2. Regulation by Energy Charge. Energy charge was defined by Atkinson (1968) as equal to one half the phosphate anhydride bonds per adenosine. Since the total anhydride phosphates would, in terms of concentration, be equal to $2 \mathrm{ATP}+\mathrm{ADP}$, the energy charge (EC) can be written mathematically as:

$$
\mathrm{EC}=\frac{\mathrm{ATP}+0.5 \mathrm{ADP}}{\mathrm{ATP}+\mathrm{ADP}+\mathrm{AMP}}
$$

The concept of energy charge as a regulatory parameter was formulated by Atkinson (1968). It stated that ATP forming systems, i.e., reactions leading to the synthesis of ATP or ATP utilizing systems, i.e., biosynthetic reactions using ATP as the energy sources, would respond to energy balance in the cell in terms of all the adenylate compounds rather than to any single adenylate compound. In the cell, the total adenylate pool (AMP + ADP + ATP) is constant over short time periods. The amount of energy stored can be considered proportional to the number of anhydride phosphate bonds per adenosine.

For an energy-regenerating enzyme system (R), the activity would be high at low charge and then fall off steeply at high charge. The opposite would be true for an energyutilizing enzyme system (U). The activity would be low at low charge and then increase rapidly at high charge (Figure 4).

There is an important relationship between the intermediary metabolites and the adenylate compounds in energy-regenerating and energy utilizing metabolic sequences. In the generation of ATP, the pools of metabolic intermediates would be diminished. Their replenishment would be linked to regeneration of ATP. This overlap of function between metabolites and adenylates has

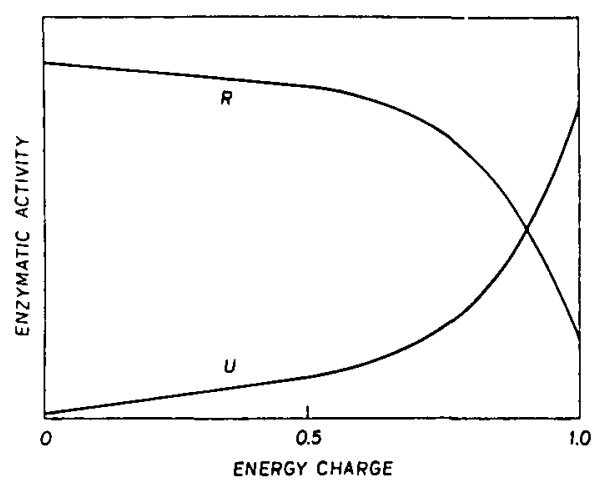

Figure 4-The enzymatic response to the adenylate energy charge for enzymes involved in regulation of ATPregenerating $(R)$ and $A$ TP-utilizing $(U)$ sequences. 
been termed amphibolic (Sanwal, 1970). It seems clear that there must be overlapping control of these overlapping functions.

Since oxidation of pyruvate via the Krebs cycle leads to the generation of ATP, it is expected that the activity of PDH is subject to regulation by the energy charge, according to Atkinson's hypothesis (R-system) (Atkinson, 1968). The activity of $\mathrm{E}$. coli PDH decreases with increasing energy charge of the adenylate pool, and this effect is maximal in the presence of acetyl CoA (Shen et al., 1968).

The activity of the mammalian pyruvate dehydrogenase multienzyme complex in vitro also depends on the level of the energy charge. An increase in the energy charge from 0.5 to 0.8 can cause a decrease of the activity of the complex by $70 \%$ (Hucho, 1974). However, the control mechanisms of PDH in mammalian systems are much more complex than what have been observed in E. coli, because mammalian PDH undergoes phosphorylation in the presence of ATP which leads to self-inactivation, and this inactivation is competitively inhibited by ADP and AMP. Various aspects of interconversion of mammalian PDH will be discussed later.

3 . Regulation by glycolytic intermediates. Shen and Atkinson (1970) showed that the activity of $\mathrm{E}$. coli PDH is stimulated by glycolytic intermediates: fructose-diphosphate, fructose-6-phosphate, glucose-6phosphate, 3-phosphoglyceric acid, phosphoenolpyruvate, dihydroxyacetone phosphate, and glyceraldehyde-3-phosphate. These stimulatory effects are exerted on the pyruvate decarboxylase component $\left(E_{1}\right)$ of the multienzyme complex. Fructose-diphosphate overcomes the inhibition resulting from a high value of the adenylate energy charge. However, Ngo and Barbeau (unpublished results) were unable to observe any significant stimulation of rat brain PDH by glycolytic intermediates. This could be explained by the evolutionary consequence of multicellular organisms. Unlike E. coli, in mammalian cells the glycolytic reactions and hence their inter- mediates occur in cytoplasm whereas the PDH system is located in the intramitochondria matrix, being physically separated from cytoplasmic compartment. It is not surprising that a different mechanism of control must have evolved in mammalian cells.

4. Regulation by enzymatic interconversion (covalent enzymatic modification). Apart from the regulation by metabolites, the mammalian PDH is also regulated by two enzymes which are both components of the multienzyme complex. A kinase inactivates the complex by phosphorylation in the presence of ATP and low $\mathrm{Mg}^{2+}$ concentration, and the reactivation is carried out by a phosphatase that dephosphorylates the phosphorylated complex in the presence of high $\mathrm{Mg}^{2+}$ concentration. The site of phosphorylation by kinase is the pyruvate decarboxylase (E1) (Reed et al., 1972). The kinase is strongly bound to the dihydrolipoamide transacetylase (E2) and the phosphatase is much more loosely bound than the kinase (Linn et al., 1969). The inactivation and reactivation by the kinase and the phosphatase are under metabolic control. Pyruvate was found to protect PDH from phosphorylation and inactivation by the kinase. This protective effcct of the pyruvate can be of physiological importance, because the reductive equivalents necessary for gluconeogenesis must be produced by oxidation by pyruvate in the mitochondria when lactate is not the starting material for gluconeogenesis. Gluconeogenesis by way of oxalacetate is possible only if part of the pyruvate is oxidized so as to provide the NADH necessary for this purpose. Pyruvate itself makes this possible by protection of the pyruvate dehydrogenase multienzyme complex against inactivation by the kinase. The Michaelis constant of ATP for kinase is low, so that in most metabolic states of the cell ATP concentration suffices to saturate the kinase. Thus, in the absence of interaction with other metabolic parameters the phosphorylation-dephosphorylation cycle is a futile cycle representing a waste of ATP. However, when one takes $\mathrm{Mg}^{2}+$ into account, then the operation of the cycle seems to make sense, since at physiological concentrations only the phosphatase fails to be saturated by magnesium ions. Thus, a variation of this concentration in vivo should influence the phosphatase activity, which in turn will regulate the activity of the whole pyruvate dehydrogenase multienzyme complex.

Brain pyruvate dehydrogenase complex was shown to be interconvertible through the phosphorylation and dephosphorylation cycle by Siess et al. (1972). Unlike the heart and kidney PDH, the brain PDH activity does not fall below $50 \%$ of the total activity upon fasting, whereas the activity of the kidney and muscle enzymes drops drastically to only about $15 \%$ of the total activity (Wieland et al., 1971; Wieland et al., 1972). PDH is an excellent example, illustrating an important principle in enzymatic regulation, i.e., the same enzyme in different tissues or organs can be regulated in a very different manner.

Blass and Lewis (1973) showed that brain PDH complex has a broad $\mathrm{pH}$ optimum around 7.8. Furthermore, they found that acetyl CoA does not inhibit the PDH activity, which is in contradiction with our results (Ngo and Barbeau, J. Neurochem. in press, 1978) and the results of Wieland et al. (1972, 1975). The rate of decarboxylation of pyruvate to $\mathrm{CO}_{2}$ and acetyl $\mathrm{CoA}$ by PDH of rat brain homogenate is very low in newborn rats, however, it increases markedly during the first postnatal month (Wilbur and Portel, 1974). The activity of brain mitochondria PDH from mature rats is initially high and does not increase with the addition of $\mathrm{Mg}^{2+}$ and $\mathrm{Ca}^{2+}$ or partially purified pyruvate dehydrogenase phosphatase or with longer incubations. The proportion of pyruvate dehydrogenase in the active form in brain changes inversely with changes in mitochondrial energy charge, whereas total pyruvate dehydrogenase activity does not change (Jope and Blass, 1975). Jope and Blass (1976) reported that treatment of mice with amobarbital and pentobarbital decreases the ac- 
tive form of PDH by $50 \%$. The proportion of $\mathrm{PDH}$ in the active form increases during ischemia. Anesthesia with amobarbital slows down the activation during ischemia, but does not alter the total amount of $\mathrm{PDH}$. The concentration and ratios of the adenylate nucleotides and the energy charge increases as the proportion of PDH in the active form decreases during ischemia. Similar results have been observed by Ksiezak (1976) in guinea pig brain. After treatment with insulin, the proportion of brain PDH in the active form increases by $30 \%$, but the energy charge does not change. Furthermore, treatment of mice with ether, morphine, ethanol or diazepam does not change the proportion of pyruvate dehydrogenase in the active form, although these treatments have been reported to alter pyruvate oxidation in brain in vivo. Thus, one can conclude that treatments which alter pyruvate oxidation in the brain do not consistently alter the proportion of $\mathrm{PDH}$ in the active form, unless they also simultaneoulsy alter the energy charge (Jope and Blass, 1976). The activity of the PDH in isolated rat liver mitochondria declines rapidly and irreversibly. This has been attributed to a limited proteolysis by some lysosomal proteolytic enzymes (Wieland, 1975). Insulin, by lowering long chain acyl-CoA in adipose tissue, leads to deinhibition of the mitochondria adenine nucleotide translocase system and thereby to a drop in the mitochondrial ATP/ADP ratio. The relative increase of ADP which inhibits the PDH kinase competitively with ATP is to shift the phosphorylation equilibrium of the PDH system towards the active dephosphorylated enzyme (Loffler et al, 1975). Isolated beef heart and rabbit heart mitochondria have also been shown to be regulated by phospho and dephosphorylation (Schuster et al., 1975; Chiang and Sacktor, 1975). The activity of the regulatory enzymes involved in the inactivation and reactivation of the pyruvate dehydrogenase multienzyme complex from isolated rat liver mitochondria may be controlled by both the intramitochondrial NADH/NAD

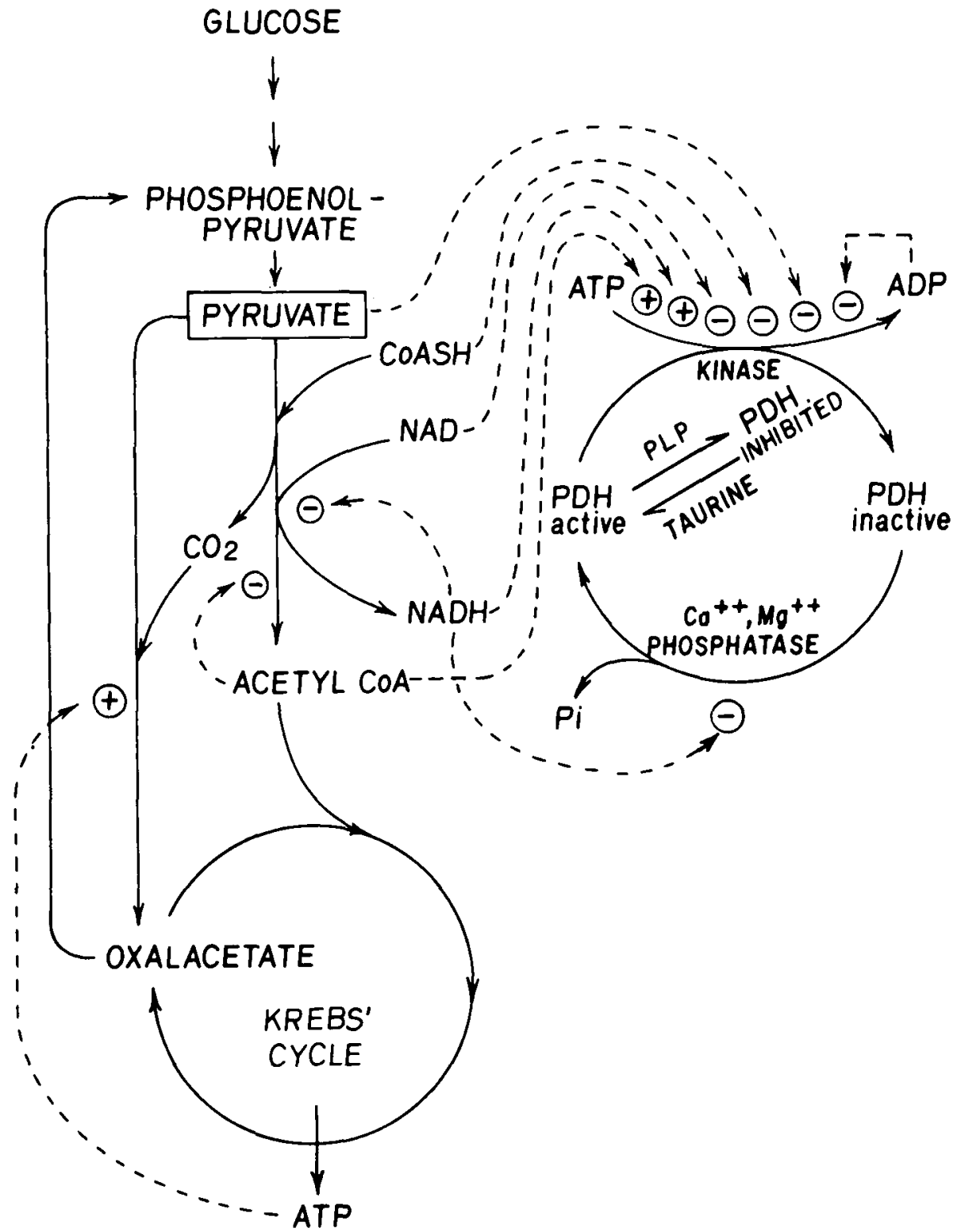

Figure 5-Diagramatic summary of the regulation of pyruvate metabolism by metabolites, neurotransmitter, metal ions and enzymatic interconversion. Solid lines indicate chemical transformation and dashed-lines indicate regulatory signal with $\Theta$ and $\oplus$ indicating inhibition and activation, respectively. $P L P=$ pyridoxal phosphate.

and acetyl CoA/CoASH ratios (Batenburg and Olson, 1975; Kerbey et al., 1976). Using purified pig heart pyruvate dehydrogenase, Cooper et al. (1975) showed that the ratios of acetyl CoA/CoASH and NADH/NAD modulate the kinase and phosphatase reactions in addition to their effects on the dehydrogenase reaction. Withdrawal of prolactin or insulin from the circulation of lactating rats leads to increased inactivation by phosphorylation of mammary gland PDH. Pro- lactin may act by priming the tissue to respond directly to normal concentration of circulating isulin and by this may be responsible for the increased activation of the enzyme during the course of normal lactation (Field and Coore, 1976). Stansbie et al. (1976) found that, in rat epididymal foot-pads, about $80 \%$ of the pyruvate dehydrogenase phosphatase was extramitochondrial in location and therefore probably not directly concerned with the regulation of PDH activity. Human heart 
PDH was also shown to be regulated by phosphorylation and dephosphorylation (Stansbie, 1976).

Batenburg and Olson (1976) recently showed that the pyruvate dehydrogenase kinase activity of a rat liver mitochondrial extract was stimulated strongly by acetyl CoA and was inhibited by NAD and CoA $\mathrm{SH}$. In contrast to acetyl CoA, octanoyl-CoA inhibited the kinase activity. Thus, the inactivation of PDH by fatty acid in isolated rat liver mitochondria may be mediated through effects of the NADH/NAD and acetyl CoA/CoASH ratios on the interconversion of the active and inactive forms of the enzyme complex catalyzed by PDH kinase and PDH phosphatase.

Figure 5 summarizes the known regulatory parameters in mammalian pyruvate metabolism. It is to be noted that the products of the PDH catalyzed reaction are not only capable of inhibiting directly the active form of the enzyme, but may also, together with substrates of the enzyme complex, regulate the PDH complex by covalent modification (Batenburg and Olson, 1976).

\section{ACKNOWLEDGMENTS}

The above studies were supported by grants from L'Association Canadienne de l'Ataxie de Friedreich and the Medical Research Council of Canada. The authors would like to thank Miss Marie Charbonneau for technical assistance and Miss Suzanne Gariépy for typing the manuscript.

\section{REFERENCES}

ATKINSON, D. E. (1968). The energy charge of the adenylate pool as a regulatory parameter. Interaction with feedback modifiers. Biochemistry, 7, 4030-4034.

BARBEAU, A. (1975). Preliminary studies on pyruvate metabolism in Friedreich's ataxia. Trans. Amer. Neurol. Ass., 100, 164-165.

BARBEAU, A., BUTTERWORTH, R. F., NGO, T., BRETON, G., MELANCON, S., SHAPCOTT, D., GEOFFROY, G. and LEMIEUX, B. (1976). Pyruvate metabolism in Friedreich's ataxia. Can. J. Neurol: Sci., 3, 379-388.

BATENBURG, J. J. and OLSON, M. S. (1976). Regulation of pyruvate dehydrogenase by fatty acid in isolated rat liver mitochondria. Biochem. Biophys. Res. Commun., 66, 533-540.

BATENBUR, J. J. and OLSON, M. S. (1976). Regulation of pyruvate dehydrogenase by fatty acid in isolated rat liver mitochondria. J. Bio. Chem., 251, 1364-1370.

BLASS, J. P., AVIGAN, J. and UHLENDORF, B. W. (1970). A defect in pyruvate decarboxylase in a child with an intermittent movement disorder. J. Clin. Invest., 49, 423-432.

BLASS, J. P., KARK, R. A. P. and MENON, N. K. (1976). Low activities of the pyruvate and oxoglutarate dehydrogenase complexes in five patients with Friedreich's ataxia. New Engl. J. Med., 295, 62-67.

BLASS, J. P. and LEWIS, C. A. (1973). Kinetic properties of the partially purified pyruvate dehydrogenase complex of ox brain. Biochem. J., 131, 31-37

CHANGEUX, J. P. (1965). The control of biochemical reactions. Scient. Amer., 212, 36-45.

CHAING, P. K. and SACKTOR, B. (1975). Control of pyruvate dehydrogenase activity in intact cardiac mitochondria. J. Biol. Chem., 250, 3399-3408.

COOPER, R. H., RANDLE, P. J. and DENTON, R. M. (1975). Stimulation of phosphorylation and inactivation of pyruvate dehydrogenase by physiological inhibitors of the pyruvate dehydrogenase. Nature, 257, 808-809.

DENTON, R. M., RANDLE, P. J., BRIDGES, B. J., COOPER, R. H., KERBEY, A. L., PARK, H. T., SEVERSON, D. L., STANBIE, D. and WHITEHOUSE, S. (1975). Regulation of mammalian pyruvate dehydrogenase. Molecular and Cellular Biochem., 9, 27-53.

FIELD, B. and COORE, H. G. (1976). Control of rat mammary gland pyruvate dehydrogenase by insulin and prolactin. Biochem. J., 156, 333-337.

HUCHO, F. (1974). Regulation of the mammalian pyruvate dehydrogenase multienzyme complex by $\mathrm{Mg}^{2+}$ and the adenine nucleotide pool. Europ. J. Biochem., 46, 499.505.

HUCHO, F. (1975). The pyruvate dehydrogenase,. Angrew, Chem. Internat. Edit., $14,591-601$.

JOPE, R. and BLASS, J. P. (1975). A comparison of the regulation of pyruvate dehydrogenase in mitochondria from rat brain and liver. Biochem. J., 150, 397-403.

JOPE, R., and BLASS J. P. (1976). The regulation of pyruvate dehydrogenase in brain in vivo. J. Neurochem., 26, 709-714.

KARK, R. A. P., BLASS, J. P. and ENGLE, W. K. (1974). Pyruvate oxidation in neuromuscular diseases. Neurology, 24, 964-971.

KERBEY, A. L., RANDLE, P. F., COOPER, R. H., WHITEHOUSE, S., PASK, H. T. and DENTON, R. M. (1976). Regulation of pyruvate dehydrogenase in rat heart. Biochem. J., 154, 327-348.

KSEIZAK, H. (1976). Effect on hypoxia, ischemia and barbiturate anesthesia on interconversion of pyruvate dehydrogenase in guinea pig brain. F.E.B.S. Letters, 63, 149-153.
LINN, T, C., PETTIT, F. H., HUCHO, F and REED, L. J. (1969), $\iota$ acid dehydrogenase complexes, XI. Comparative studies of regulatory properties of the pyruvate dehydrogenase complexes from kidney, heart and liver mitochondria. Proc. Nat. Acad. Sci. (Wash.) 64-227-234.

LOFFLER, G., BARD, S. and WIELAND, O. H. (1975). Control of pyruvate dehydrogenase interconversion by palmitoylCoenzyme $A$ as related to adenine nucleotide translocation in isolated fat cell mitochondria. F.E.B.S. Letters, 60 , 269-274.

MCILWAIN, H. and BACHELARD, H. S. (1971). Biochemistry and the central nervous system. 4th Edition, Baltimore, Williams and Wilkins.

REED, L. J. (1969). Pyruvate dehydrogenase complex. In: Current Topics in Cellular Regulation ( B. L. Horecker and E. R. stadtman, editors), Volume 1, Academic Press, New York, pp. 233-251.

REED, L. J. (1974). Multienzyme complexes. In: Accounts of Chemical Research, Vol. 7, pp. 40-46.

REED, L. J., LINN T. C., HUCHO, F., NAMIHIRA, G., BARRERA, B. R., ROCHE, T. E., PELLEY, J. W. and RANDALL, D. D. (1972). Molecular aspects of regulation of the mammalian pyruvate dehydrogenase complex. In: Metabolic Interconversion of Enzymes. (Wieland, O., Heimreich, E. and Holzer, H., editors). Springer-Verlag, Berlin, pp. 281-291.

REYNOLDS, S. F. and BLASS J. P. (1976). A possible mechanism for selective cerebellar damage in partial pyruvate dehydrogenase deficiency. Neurology, 26, 625-628.

SANWAL, B. D. (1970). Allosteric controls of amphibolic pathways in bacteria. Bact. Rev., 34, 20-39.

SCHIMKE, R. T. (1969). On the role of synthesis and degradation in regulation of enzyme levels in mammalian tissues. In: Current Topics in Cellular Regulation, (B. L. Horecker and E. R. Stadtman, editors). Vol. 1, Academic Press, New York, pp. 77-124.

SCHUSTER, S. M., OLSON, M. S. and ROUTH, C. A. (1975). Studies on the regulation of pyruvate dehydrogenase in isolated beef heart mitochondria. Arch. Biochem. Biophys., 171, 745-752.

SHEN, L. C. and ATKINSON, D. E. (1970). Regulation of pyruvate dehydrogenase from Escherichic coli. Interaction of adenylate energy charge and other regulatory parameters. J. Biol. Chem., 245, 5974-5978.

SHEN, L. C., FALL, L., WALTON, G. M. and ATKINSON, D. E. (1968). Interaction between energy charge and metabolite modulation in the regulation of enzymes of amphibolic sequences. Phosphofructokinase and pyruvate dehydrogenase. Biochemistry, 7, 4041-4045.

SIESS, E., WITTMANN, J. and WIELAND, O. (1971). Interconversion and 
kinetics properties of pyruvate dehydrogenase from brain. Hoppe Seylers $Z$. Physiol. Chem., 352, 447-452.

SIESS, E., WITTMANN, J. and WIELAND, O. (1972). Interconversion and kinetic properties of pyruvate dehydrogenase from brain. Hoppe-Seylers $\mathrm{Z}$. Physiol. Chem., 352, 447-452.

STANSBIE, D. (1976). Regulation of the human pyruvate dehydrogenase complex. Clin: Sci. Molec. Med., 51, 445-452.

STANSBIE, D., DENTON, R. M., BRIDGES, B. J., PASK, H. T. and RANDLE, P. J. (1976). Regulation of pyruvate dehydrogenase and pyruvate dehydrogenase phosphate phosphatase activity in rat epidedymal fat-pads. Biochem. J., 154, $225-236$

TSAI, C. S., BURGETT, M. W. and REED L. J. (1973). $\alpha$-keto acid dehydrogenase complex. J. Biol. Chem., 248, 8348-8352.

WIELAND, O. H. (1975). On the mechanism of irreversible pyruvate dehydrogenase inactivation in liver mitochondria extracts. F.E.B.S. Letters, 52, 44-47.

WIELAND, O., SIESS, E., FUNCKE, H. J. V., PATZELT, C., SCHIRMAN, A., LOFFLER, G. and WEISS L. (1972). Regulation of the mammalian pyruvate dehydroegnase complex: Physiological aspects and characterization of PDH-phosphatase from pig heart. In: Metabolic Interconversion of Enzymes, (Wieland, O., Helmreich, E. and Holzer, H., editors). SpringerVerlag, Berlin, pp. 293-309.

WIELAND, O., SIESS, E., SCHULZEWETHMAR, F. H., FUNCKE, H. G. V.and WINTON, B. (1971). Active and inactive forms of pyruvate dehydrogenase in rat heart and kidney: Effect of diabetes, fasting, and refeeding on pyruvate dehydrogenase interconversion. Arch. Biochem. Biophys., 143, 593-601.

WILBUR, D. O. and PORTEL, M. S. (1974). Development of mitochondrial pyruvate metabolism in rat brain. J. Neurochem., 22, 709-715. 